\title{
Modern Technologies for Micro-drilling of the Fuel Injector Nozzle used in Motor Vehicles - A Review of the Literature
}

\author{
Dorinel Popa ${ }^{1, *}$, and Cristin-Olimpiu Morariu ${ }^{2}$ \\ ${ }^{1}$ Transilvania University of Brașov, Department of Manufacturing Engineering, Mihai Viteazu 5, \\ 500174, Romania, \\ ${ }^{2}$ Transilvania University of Braşov, Department of Manufacturing Engineering, Mihai Viteazu 5, \\ 500174, Romania
}

\begin{abstract}
To cope with the pollution norms and an improvement of the combustion of the internal combustion engines, high-quality holes with diameters smaller than $145 \mu \mathrm{m}$ are needed for the manufacture of fuel injection nozzles. The current practice of using drilling by electro-discharge machining of fuel injection nozzles is limited in terms of the size of the hole it can efficiently produce and the time required for drilling. In addition, the cost of the tool is high. This paper presents an investigation into a sequential laser and electro-discharge micro-drilling technique for the manufacture of fuel injection nozzles. A pilot hole drilled with a laser is removed by electrodischarge. It was found that this hybrid process eliminated the problems of reformed and heat-affected areas usually associated with the laser drilling process. The new process has allowed a reduction in total drilling time compared to standard electro-discharge machining drilling, as less material is removed from the electro-discharge machining. The quality of the holes is as good as direct electro-discharge machining drilling. This technique has allowed valuable cost savings and increased production capacity for the manufacture of the fuel injector nozzle.
\end{abstract}

\section{Introduction}

The manufacturing process comprises all mechanical, physical and chemical applications through which the geometry, shape and/or properties of raw material are converted into finished products. The manufacturing process involves activities such as product design, raw material selection, production process planning, quality assurance, raw material management and marketing. Over the centuries materials and processes have been gradually refined, using new materials and more complex operations to increase production and quality.

Taking into account the increasing requirements for lightweight, multifunctional and high-density products, more lines and holes are needed in an increasingly limited space at the same time as improved quality. To achieve these objectives, the research media have made a continuous improvement of manufacturing technologies, namely the performance of micro-

*Corresponding author:popa.dorinel71@yahoo.com 
drilling, methods, mechanical properties, as well as the quality and accuracy of holes, the process of chip formation and their removal. In parallel, it has been and is necessary to improve the operating conditions of the means of production to increase the dimensional accuracy and the quality of the drilled surfaces.

The current requirements for increasing energy efficiency and reducing pollutant emissions into the atmosphere require injection nozzles with smaller and smaller spray holes, respectively with a diameter of less than $145 \mu \mathrm{m}$ on a reduced thickness of $1 \mathrm{~mm}$ of alloy steels. These requirements can be solved by micro-drilling technologies through which holes with small diameters between $1 \mu \mathrm{m}$ and $1 \mathrm{~mm}$ are drilled.

In modern applications, unconventional micro-drilling is used which unlike conventional micro-drilling using a drill and which has limits on drilling diameter, this unconventional technology uses various means of operation, namely mechanical, electrical, chemical, thermal or combinations thereof.

Among the unconventional micro-drilling technologies respectively laser, electric discharge processing, electron beams, electro-chemistry and spark-assisted chemical engraving, laser drilling technology is the most popular, having advantages but also disadvantages in terms of cost, accuracy processing as well as the minimum achievable dimensions.

\section{Laser micro-drilling}

Laser micro-drilling technology is a processing technique that uses a controlled ablation mechanism. Figure 1 shows the diagram of the laser micro-drilling process. With this technology, holes with a diameter of one micron to several hundred microns are made. Laser micro-drilling technology is influenced by the characteristic characteristics of the material (composition and thickness) and the laser (frequency, wavelength and energy density).

In principle, laser micro-drilling is performed in two phases:

-photo-chemistry,

-photo-thermal.

In the first phase, the energy of the laser photon falls directly on the surface of the material to be processed, breaking the connections, which determines the evacuation of the material from the volume under illumination.

In the second phase, the temperature exceeds the melting point because the material absorbs the laser energy, leading to the removal of the material by evaporation [1-5].
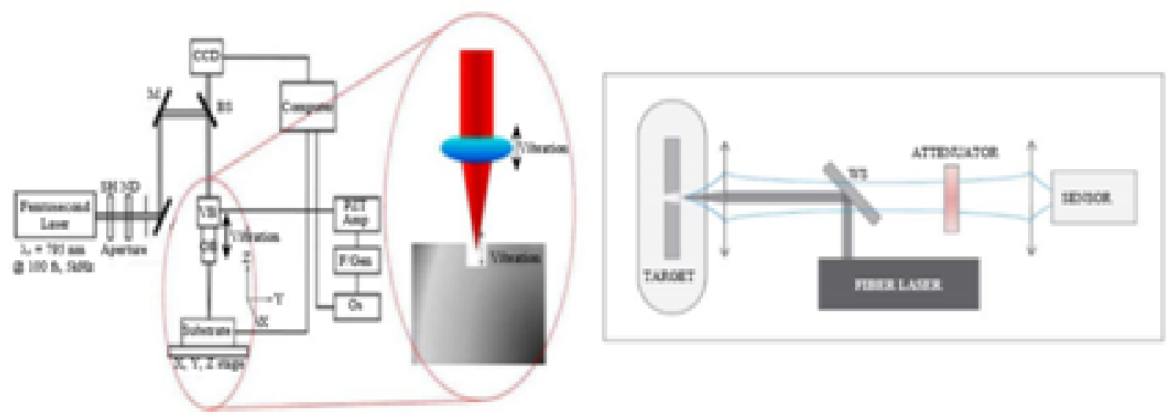

Fig.1. Laser micro-drilling scheme [6-7]. 


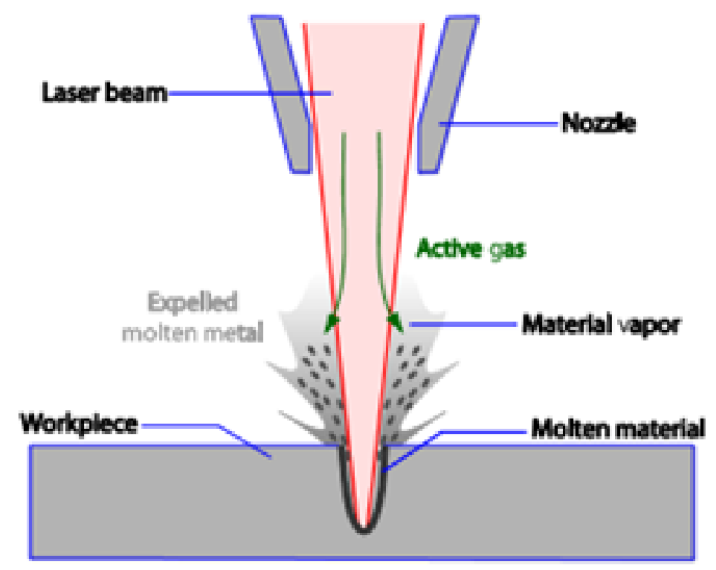

Fig.2. Laser drilling works [8].

Figure 2 shows the basic elements of a laser pulse that strikes the surface of a workpiece.

To analyse the feasibility of using laser energy for micro-drilling, the researchers used various technologies, taking into account numerous factors, including laser type, system development and analytical modelling of the finite element method, elementary hole composition, process optimization, machining underwater laser, the duration of the drilling process and the energy corresponding to the required process and the reduction of manufacturing costs.

After the discovery of the laser effect, a very large number of coherent radiation sources were manufactured. Lasers can be divided into several categories: according to the mode of operation (continuous and pulse), according to the nature of the active medium used (lasers with solid, liquid and gas), according to the spectral range in which they emit, etc. Also, lasers can be divided according to the electronic nature involved in obtaining laser radiation: lasers with bound electrons (ribbon laser, $\mathrm{Nd}^{3+}$ doped glass laser, laser $\mathrm{Ti}$ : sapphire, semiconductor lasers, $\mathrm{CO}_{2}$ laser, ionic, with metallic, chemical, vibronic vapours and free electron lasers, respectively. Of these, several used in industry are those with bound electrons [6-8].

The durations for laser pulse ablation are of the order $\mathrm{n}\left(10^{-9}\right), \mathrm{p}\left(10^{-12}\right)$ şi $\mathrm{f}\left(10^{-15}\right)$ [9-11].

Analysing unconventional drilling technologies, we found that laser micro-drilling is most used for processing parts with complex, hard and difficult to drill by other methods, such as ceramics, glass and composite materials.

\section{EDM micro-drilling}

EDM micro-drilling is a complex process that is performed by the simultaneous action of thermal, mechanical, electrical, and chemical operations (Figure 3.). In this technology, with the help of electricity, discrete high-frequency sparks are created, which remove the material through the thermal energy produced by sparks that are generated by two electrodes that are very close. Sparks are produced in a dielectric medium when a large potential difference is applied between the electrodes. Following this process, the area between the electrodes reaches a very high temperature, the material melts and then evaporates. The molten material in the space between the electrodes is solidified and transported of a dielectric liquid circulating at high pressure through the processing gap, in the form of particles (residues) $[12,13-16]$. 

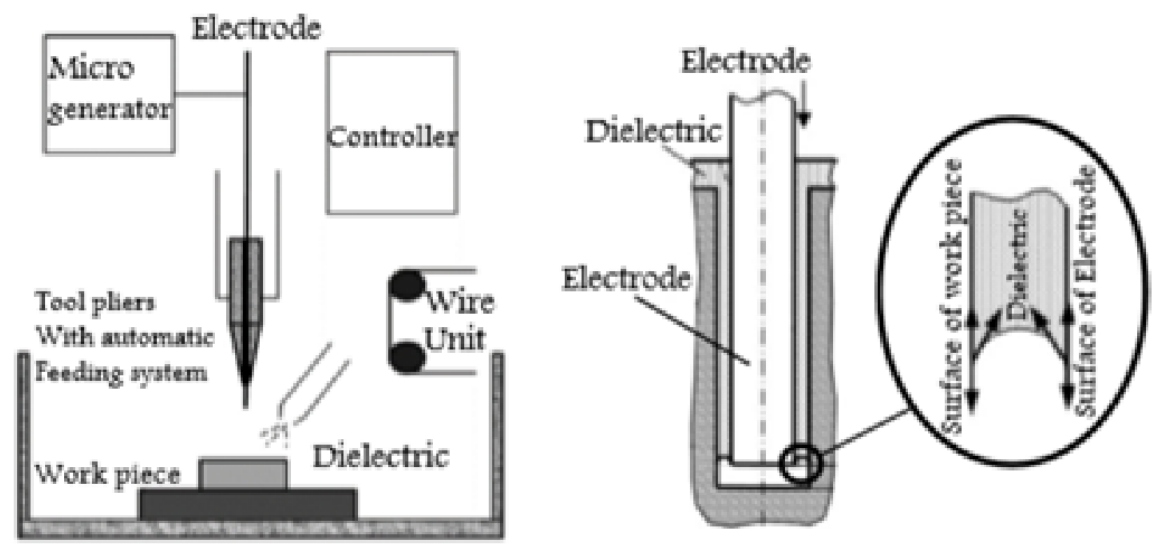

Fig.3. Schematic diagram of EDM [17,18].

Drilling with EDM is a micro-electric discharge processing process that uses a microwire as an electrode, which copies the mirror image into the part to be drilled, being used for processing parts with complex shapes and difficult to process (tool steels, aerospace alloys and toilets).

The electrodes used for micro-drilling with EDM can be made of copper-tungsten and graphite using different technologies.

Through this micro-drilling technology, holes are made that have a high aspect ratio, compared to conventional manufacturing technologies. Based on this technology, a series of researches were made regarding the realization of micro-holes with a very small diameter under $1 \mu \mathrm{m}$. Good results were obtained by using electrodes made of silicon [19].

\section{Sequential micro-drilling with Laser and EDM}

To improve the combustion of an internal combustion engine and reduce emissions into the atmosphere, high-quality holes with diameters smaller than $140 \mu \mathrm{m}$ are required for fuel injector nozzles. Drilling using the electro-discharge (EDM) method of injector nozzles is limited in terms of the size of the holes it produces and the duration of the drilling process. Also, the tool costs are high. The sequential micro-drilling technique laser-EDM for making spray nozzles of fuel injection nozzles is a technique that eliminates the disadvantages of using laser and EDM processing. A pilot hole made using laser technology is adjusted by drilling with EDM. Sequential processing has been found to eliminate reformed problems as well as those due to the specific temperature of laser processing. This machining process reduces the total drilling time because the amount of material is smaller. Higher quality holes are made and no more holes need to be ground. This technique allows to increase productivity and reduce production costs.

Figure 4 illustrates the combined hole with laser and EDM detailing the diameters of the holes made by laser, the size of the area affected by heat and finally the diameter of the hole made by EDM. 


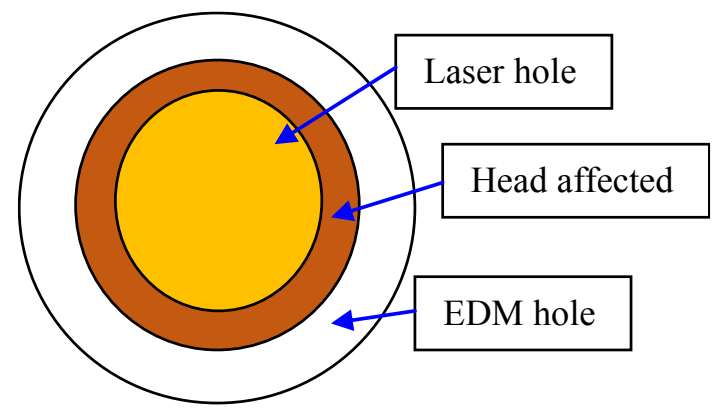

Fig. 4. Illustration of sequential laser and EDM drilling.

With the help of the laser, holes with positive and negative conical shape as well as parallel holes can be made. [20]

Studies have shown that to make a hole through EDM, the time period is reduced by $70 \%$.

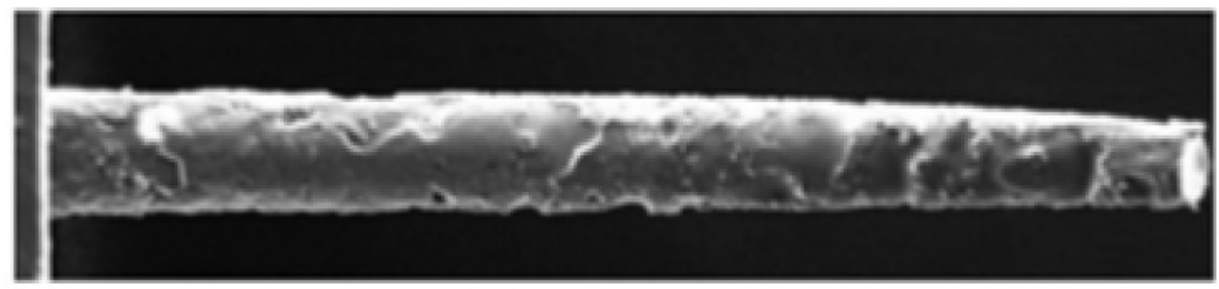

$\mathrm{a}$

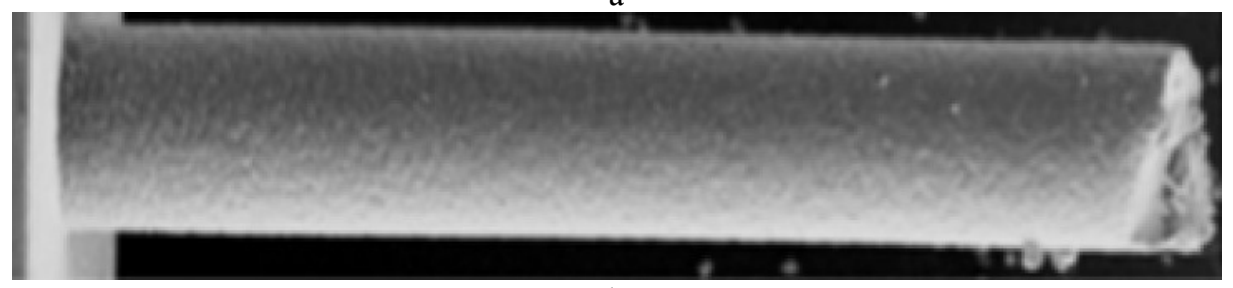

b

Fig. 5. SEM image of a plastic footprint of a hole made with: a- laser; b- laser-EDM after drilling EDM. [20].

Figure 5a shows a plastic footprint that was taken from a laser machined hole.

Figure $5 \mathrm{~b}$ shows a significant improvement in the quality of the hole made by laser-EDM. Analysing figure $6 \mathrm{a}$, in which is presented the image of the section of the hole made with EDM and figure $6 \mathrm{~b}$ which illustrates the section of the hole made with laser and EDM it is found that through the sequential technology holes are produced that have a comparable quality with holes made by EDM. In both processing processes, a process of cleaning the walls of the holes made is required. 


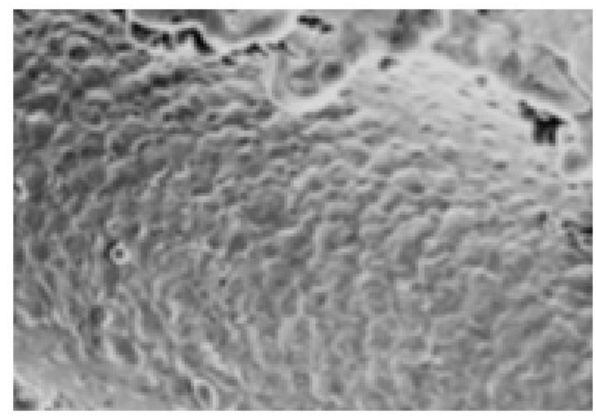

a

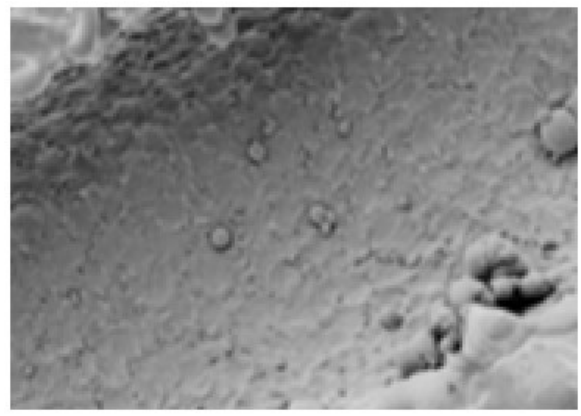

b

Fig. 6. Image of a sectioned hole made by: a- standard EDM technology; b- laser-EDM technology [20].

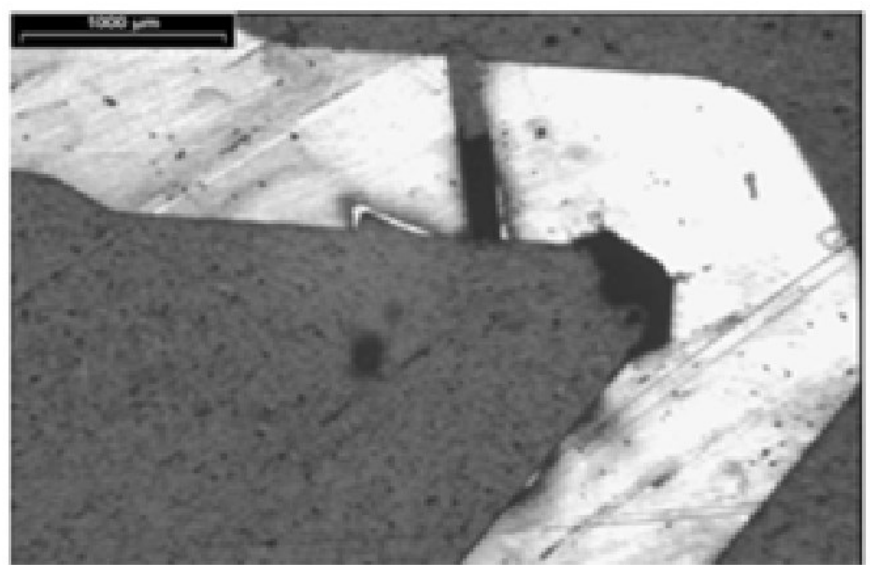

Fig. 7. Fuel nozzle-section - laser-drilled EDM. [20]

For the fuel injector and therefore the nozzle to operate at normal parameters, a critical problem may arise when aligning the laser-EDM hole, as spraying the fuel nozzle influences the combustion of fuel in the combustion chamber, having implications for internal combustion engine performance (fig.7.).

Figure 8 shows a section of the nozzle of the respective fuel injector of the fuel spray orifices. 


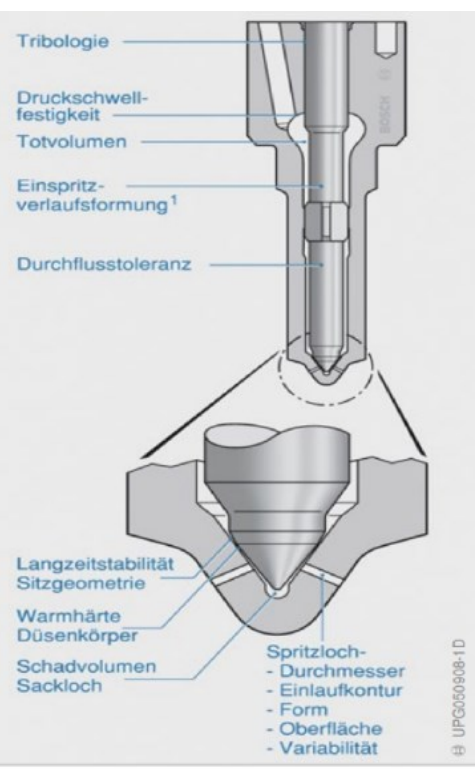

Fig. 8. Schematic view of the fuel injector nozzle [21].

\section{Discussion}

There are a several of advantages to choosing this processing method for fuel injector nozzle holes. A major advantage is that it reduces the cost of processing mainly by reducing processing time and reducing labour costs. Production capacity can be increased because it will take less time to process. Therefore, no additional investment will be needed to increase productivity.

\section{Conclusions}

Analysing the presented research, we can say that to reduce manufacturing costs and increase productivity, the new laser-EDM drilling technique makes holes comparable to standard EDM drilling. To meet the increasingly severe pollution conditions, the size of the nozzle holes can be reduced, thus making the fuel as fine as possible. Compared to standard EDM drilling during laser-EDM drilling, a smaller radial offset is obtained because the flow of dielectric fluid and debris is discharged through the pilot holes during drilling, thus reducing drilling time and increasing drilling efficiency.

Of all the unconventional techniques, laser micro-drilling is the most widely used due to its high drilling speed, but has the disadvantage of poor surface finishing. To solve these difficulties, sequential processing or laser-EDM micro-drilling must be used.

In conclusion, for the selection of appropriate technology, for micro-drilling it is necessary to understand the available technologies, as well as factors such as the quality of the processed surfaces, the cost and duration of the drilling process.

\section{References}

1. H. Huang, L.M. Yang, and J. Liu, Laser Applications in Microelectronic and Optoelectronic Manufacturing (Lamom) Xviii, 8607 (2013). 
2. S. Broude, Industrial Laser Solutions for Manufacturing, TRUMPF Industrial Laser Technology: New Hampshire, USA ( 2012).

3. R. Biswas, A.S. Kuar, and S. Mitra, Journal of Materials Research and Technology, 4(3), 323 (2015)

4. H.-N. Jia, et al., Chinese Physics Letters, 30(4), 044202 (2013).

5. R. Witte, et al. Advanced Laser Technologies (2008)

6. F.P. Mezzapesa, et al., Physics Procedia, 41, 670 (2013)

7. J.K. Park, et al., Transactions of Nonferrous Metals Society of China, 22, S801 (2012)

8. A. Alzaydi, Optical Engineering, 57(12), 120901 (2018)

9. T. Dreischuh, et al., Laser Beam Drilling Efficiency unit: the. 2010. 7751, 77511H(2010).

10. J. Tu, et al., Optics and Lasers in Engineering, 55: p. 275 (2014).

11. C.-H. Tsai, and C.-C. Li, Journal of Materials Processing Technology, 209(6), 2838 (2009).

12. G. D'Urso, G. Maccarini, and C. Merla, Sheet Metal 2013, 549, 503 (2013).

13. H. Tonget al., Precision Engineering, 37(1), 213 (2013).

14. Y. Yahagi, et al., 5th CIRP Conference on High Performance Cutting 2012, Zurich (2012).

15. H. Tong, Y. Li, and L. Zhang, Manufacturing Engineering and Automation Ii, 1-3, 591 (2012).

16. Jahan, M.P., Y.S. Wong, and M. Rahman, Journal of Manufacturing Processes, 1 4(3), 343 (2012).

17. J. Li, et al., Journal of Mechanical Science and Technology, 27(1), 185 (2013).

18. G. D'Urso, G. Maccarini, and C. Ravasio, International Journal of Advanced Manufacturing Technology, 72(9-12), 1287 (2014).

19. K. Egashira, Y. Morita, and Y. Hattori, Precision Engineering, 34(1), 139 (2010).

20. Lin Li, C. Diver, J. Atkinson, R. Giedl-Wagner, H. J. Helml, Annals of the CIRP Vol. 55, 1(2006)

21. Konrad Reif (Hrsg.). Dieselmotor-Management Systeme, Komponenten, Steuerung und Regelung 6., Aufage, Springer Vieweg, Wiesbaden, p. 193 (2020) 